\title{
Unusual Extramedullary Relapse after Haploidentical Bone Marrow Transplantation in a Patient with Acute Lymphoblastic Leukemiat Yasuhiro Ebihara ${ }^{1,2 *}$, Shohei Yamamoto ${ }^{1}$, Shinji Mochizuki ${ }^{1,2}$, Masao Tsukada ${ }^{3}$, Yuki Taya ${ }^{3}$, Aki Sato $^{3}$, Toshiro Kawakita ${ }^{3}$, Seiko Kato $^{3}$, Jun $\mathrm{Ooi}^{3}$, Satoshi Takahashi ${ }^{3}$, Arinobu Tojo ${ }^{3}$ and Kohichiro Tsuji ${ }^{1,2,4}$
}

${ }^{1}$ Department of Pediatric Hematology/Oncology, Research Hospital, The Institute of Medical Science, The University of Tokyo, Tokyo, Japan ${ }^{2}$ Division of Stem Cell Processing, Center for Stem Cell Biology and Regenerative Medicine, The Institute of Medical Science, The University of Tokyo, Tokyo, Japan ${ }^{3}$ Department of Hematology/Oncology, Research Hospital, The Institute of Medical Science, The University of Tokyo, Tokyo, Japan

${ }^{4}$ National Hospital Organization, Shinshu Ueda Medical Center, Ueda, Japan

\begin{abstract}
Extramedullary relapse of acute lymphoblastic leukemia (ALL) after allogeneic hematopoietic stem cell transplantation (HSCT) is infrequent. In general, the sites of extramedullary relapse are mainly the central nervous system (CNS) and testis, which are so called sanctuary sites. In sites of extramedullary relapse, renal or pancreatic involvements after HSCT are extremely rare in ALL. Here we described a case of 17 year-old male, who was diagnosed as ALL with the $t(1 ; 19) / T C F 3-P B X 1$. At twenty months after the fourth myeloablative HSCT, he had extramedullay relapse, which concurrently occurred in pancreas and bilateral kidneys in spite of maintaining hematological complete remission in bone marrow (BM). He had never had extramedullary replaspe including in CNS and testis before this episode. Concurrent involvement of two solid organs (pancreas and kidney) after HSCT as in the present case has not been described.
\end{abstract}

Keywords: Acute lymphoblastic leukemia; Extramedullary relapse; Haploidentical bone marrow transplantation

\section{Introduction}

Extramedullary relapse of acute lymphoblastic leukemia (ALL) after allogeneic hematopoietic stem cell transplantation (HSCT) is infrequent with rates of extramedullary involvement reported in $11 \%$ [1]. In general, the sites of extramedullary relapse are mainly the central nervous system (CNS) and testis, which are so called sanctuary sites, that are protected from systemic chemotherapy by biological blood barriers (CNS) or distinct growth condition (lower temperature, testis) [2]. In sites of extramedullary relapse, either renal involvement or pancreatic infiltration of leukemic cells after HSCT is very rare. Concurrent involvement of two solid organs after HSCT has not been described.

\section{Case Report}

A 17-year-old male was diagnosed with acute lymphoblastic leukemia (ALL; chimeric transcription factor 3 (TCF3)/pre-B cell leukemia homobox 1 (PBX1) fusion transcript+) in October, 2006. He underwent three rounds of myeloablative allogeneic CBT to treat $\mathrm{BM}$ relapse. He suffered a third BM relapse 6 months after the fourth cord blood transplantation (CBT). He was treated with multidrug chemotherapy, but did not achieve hematological complete remission (CR) in the bone marrow (BM). Because his physical condition and level of organ tolerance were both not so damaged (even after three rounds of myeloablative CBT), we performed HSCT in November, 2010, using T cell-repleted haploidentical BM cells donated by his mother. The patient was treated with low-dose cytarabin and etoposide before receiving a myeloablative condition in gregimen (fuldarabine 25 $\mathrm{mg} / \mathrm{m}^{2} \times 4$, etoposide $30 \mathrm{mg} / \mathrm{kg} \times 1$, and cyclophosphamide $60 \mathrm{mg} / \mathrm{kg} \times 2$ ) prior to transplantation. However, an increased number of leukemic cells and chimeric TCF3/PBX1 fusion transcripts were detected in the BM 2 months later; therefore, the immunosuppressants (prednisolone; PSL and tacrolimus) were tapered rapidly and discontinued. The patient then received a donor lymphocyte infusion (DLI; $5 \times 10^{6} / \mathrm{kg} \mathrm{CD} 3+$ cells from his mother). After the DLI, he achieved molecular remission and received PSL to control graft-versus-host disease (GVHD). Chimeric
TCF3/PBX1 fusion transcripts were detected in the BM 6 months after the DLI, despite hematological CR. Therefore, we tried to taper the PSL to suppress the progression of GVHD. However, he repeated the exacerbation of interstitial pneumonitis due to chronic GVHD when PSL was decreased. Twenty months after the haploidentical BMT the patient complained of severe abdominal pain. Complete blood picture showed leukocyte count, $13,450 / \mu \mathrm{L}$ with $0 \%$ blasts, hemoglobin, 13.5 $\mathrm{g} / \mathrm{dL}$, and platelet count, $123,000 / \mu \mathrm{L}$. Serum creatinine and amylase levels were within normal limit, serum lactate dehydrogenase was markedly elevated (1,505 U/L; normal, 106 to $221 \mathrm{U} / \mathrm{L})$. Urinalysis showed no proteinuria, hematuria, or pyuria. Urine culture detected neither bacteria nor fungus. Abdominal computed tomography (CT) revealed enlargement of the pancreas with hypo-attenuating mass lesions (Figure 1a), and bilateral nephromegaly (left > right) with multiple, nodular hypo-attenuating cortical masses (Figure 1b). No mass lesions were detected on the abdominal CT performed 3 months earlier. Scintigraphy with ${ }^{67} \mathrm{Ga}$ citrate showed diffuse accumulations in the tail of the pancreas and in the left kidney (Figure 1c). No increase in the number of leukemic cells was evident in BM by flow cytometric and fluorescence in situ hybridization (FISH) analysis, although amplified chimeric TCF3/PBX1fusion transcripts were detected. Since there was no evidence of infection, the patient was diagnosed with extramedullary relapse. No CNS or testicular involvement was detected.

*Corresponding author: Yasuhiro Ebihara, Department of Pediatric Hematology-Oncology, Research Hospital, Institute of Medical Science, University of Tokyo, 4-6-1, Shirokanedai, Minato-ku, Tokyo 108-8639, Japan, Tel: 81-3-5449-5397; Fax: 81-3-5449-5428; E-mail: ebihara@ims.u-tokyo.ac.jp

Received July 19, 2013; Accepted September 12, 2013; Published September 16, 2013

Citation: Ebihara Y, Yamamoto S, Mochizuki S, Tsukada M, Taya Y (2013) Unusual Extramedullary Relapse after Haploidentical Bone Marrow Transplantation in a Patient with Acute Lymphoblastic Leukemia. J Blood Disorders Transf 4: 155. doi: 10.4172/2155-9864.1000155

Copyright: @ 2013 Ebihara Y, et al. This is an open-access article distributed under the terms of the Creative Commons Attribution License, which permits unrestricted use, distribution, and reproduction in any medium, provided the original author and source are credited. 
Citation: Ebihara Y, Yamamoto S, Mochizuki S, Tsukada M, Taya Y (2013) Unusual Extramedullary Relapse after Haploidentical Bone Marrow Transplantation in a Patient with Acute Lymphoblastic Leukemia. J Blood Disorders Transf 4: 155. doi: 10.4172/2155-9864.1000155

Page 2 of 3

Daily administration of prednisolone and weekly administration of vincristine (VCR) were started to reduce the mass lesions and clinical symptoms. Two months later, the extramedullary lesions showed a significant reduction in size (Figures $2 \mathrm{a}$ and $2 \mathrm{~b}$ ) and hematological CR in $\mathrm{BM}$ was maintained. His performance status had been kept in grade 1. Unfortunately, 3 months after the extramedullary relapse, the patient suddenly died of septic shock due to Klebsiella pneumoniae infection. Autopsy was not done.

\section{Discussion}

The results from several large-scale studies indicate that 20 $25 \%$ of patients with ALL relapse might achieve remission after immunoadoptive therapy, including DLI, after allogeneic HSCT [3-5]. The current patient received three rounds of CBT before BMT, and BM relapse occurred within 6-8 months of each round. Therefore, we performed haploidentical BMT. Although molecular relapse occurred 2 months later, an immediate DLI achieved molecular remission in the $\mathrm{BM}$, and maintained cytological remission for 21 months. ALL with the $\mathrm{t}(1 ; 19) / \mathrm{TCF} 3-\mathrm{PBX} 1$ is reported to have higher incidence for CNS

a

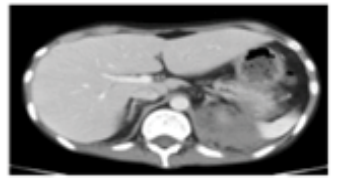

b

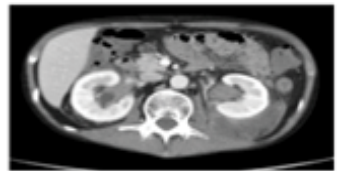

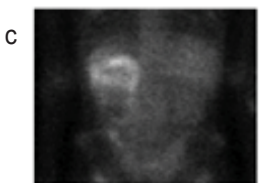

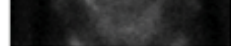
extramedullary relapse. Abdominal CT scan showed the enlargement of pancreas with hypoattenuating mass lesions (a) and bilateral nephromegaly (left > right) with multiple, nodular hypoattenuating cortical masses (b). $67 \mathrm{Ga}$ scintigraphy showed diffuse accumulation in the tail of pancreas and left kidney (c).

a

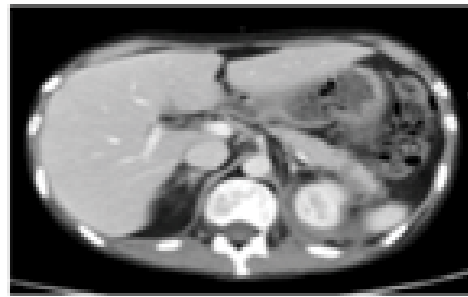

b

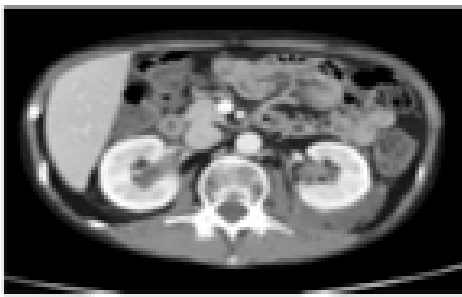

Abdominal CT scan showed hypoattenuating mass at pancreas (a) and multiple nodular hypoattenuating cortical masses around bilateral kidneys (b) were significantly reduced in size.

Figure 2: Abdominal CT scan two months after the treatment. involvement [6]. CNS and testicular relapses never occurred in this patient. Extramedullary relapse developed 18 months after the DLI, despite continued hematological CR in the BM. Thus, DLI after HSCT prevented BM relapse, but not extramedullary relapse.

Extramedullary relapse of ALL after allogeneic HSCT occurs in 11\% of cases [1]. The CNS and testes are the main sites of extramedullary relapse. These so-called "sanctuary sites" are protected from the effects of systemic chemotherapy by biological blood barriers (in the case of the CNS) or distinct growth conditions (e.g., the lower temperature in the testis). However, an unusual extramedullary involvement of leukemic cells was observed in the current patient. Renal involvement of leukemic cells after HSCT is an uncommon feature of extramedullary relapse in ALL, and only six cases have been reported [7-11]. Pancreatic infiltration by leukemic cells after HSCT is very rare, with only one case reported to date [12]. Concurrent involvement of both organs after HSCT has never been described. Like the sanctuary sites, it is possible that these organs are protected from the various anti-leukemic therapies by some biological effects [2]. Indeed, the kidney tubulo-interstitiumis reported to be a site of immune privilege, which is not fully accessible to cytotoxic T lymphocytes [13]. Therefore, the unusual extramedullary relapse observed in our patient might indicate the presence of specific biological features of his leukemic cells to infiltrate into the kidney and pancreas, such as receptors or adhesion molecules, on his leukemic cells that have high affinity for these organs.

Extramedullary relapse in ALL usually precedes BM relapse. There are two possible explanations for this: 1) the recruitment of activated cytotoxic $\mathrm{T}$ lymphocytes to leukemic organs is not fully operative or is delayed; 2) some types of leukemic cell have a high affinity for extramedullary sites, possibly due to the presence of specific receptors or adhesion molecules. For example, high expression of CXCR4 (a chemokine receptor important for the extravasation and trafficking of lymphocytes) by leukemic cells predicts extramedullary organ infiltration in childhood ALL [14]. Interestingly, some ALL patients treated with allogeneic HSCT undergo extramedullary relapse after DLI, but with a longer time lag between extramedullary and BM relapse than that observed in non-transplanted patients (who frequently experience BM relapse soon after extramedullary relapse) [11]. The current patient maintained hematological CR in the BM for 21 months after DLI. Although he died of infection 3 months after extramedullary relapse, he still maintained hematological CR in the BM. The continued hematological $\mathrm{CR}$ in the $\mathrm{BM}$ might be due to the relatively specific activity of the infused donor $\mathrm{T}$ cells within the hematopoietic tissues. DLI may trigger the recruitment of activated cytotoxic T lymphocytes, which suppress the growth of residual leukemic cells at medullary sites; however, the anti-leukemic effects of activated cytotoxic T lymphocytes within extramedullary sites might not be sufficient to inhibit the growth of residual leukemic cells.

In conclusion, the current patient showed an unusual pattern of extramedullary relapse, involving both the kidney and the pancreas, 18 months after DLI while maintaining hematological CR in the BM. This case suggests that alloimmune intervention may have an effective and long-lasting anti-leukemic effect at medullary sites. However, the anti-leukemic effects of donor leukocytes at extramedullary sites may be limited, and some types of leukemic cell might have a high affinity for specific organs that are protected by biological barriers. Further investigations are necessary to clarify the anti-leukemic effects of DLI after HSCT and the relationship between the characteristics of leukemic cells and their preferred sites of extramurally infiltration. 
Citation: Ebihara Y, Yamamoto S, Mochizuki S, Tsukada M, Taya Y (2013) Unusual Extramedullary Relapse after Haploidentical Bone Marrow Transplantation in a Patient with Acute Lymphoblastic Leukemia. J Blood Disorders Transf 4: 155. doi: 10.4172/2155-9864.1000155

Page 3 of 3

\section{References}

1. Lee KH, Lee JH, Kim S, Lee JS, Kim SH, et al. (2000) High frequency of extramedullary relapse of acute leukemia after allogeneic bone marrow transplantation. Bone Marrow Transplant 26: 147-152.

2. Goldberg SL, Mangan KF, Klumpp TR, Cropper TM, Schnall SF, et al. (1994) Lack of a graft-versus-leukemia effect in an immunologically privileged sanctuary site. Bone Marrow Transplant 14: 180-181.

3. Collins RH Jr, Shpilberg O, Drobyski WR, Porter DL, Giralt S, et al. (1997) Donor leukocyte infusions in 140 patients with relapsed malignancy after allogeneic bone marrow transplantation. J Clin Oncol 15: 433-444.

4. Bader P, Kreyenberg H, Hoelle W, Dueckers G, Handgretinger R, et al. (2004) Increasing mixed chimerism is an important prognostic factor for unfavorable outcome in children with acute lymphoblastic leukemia after allogeneic stemcell transplantation: possible role for pre-emptive immunotherapy? J Clin Oncol 22: $1696-1705$

5. Shiobara S, Nakao S, Ueda M, Yamazaki H, Takahashi S, et al. (2000) Donor leukocyte infusion for Japanese patients with relapsed leukemia after allogeneic bone marrow transplantation: lower incidence of acute graft-versushost disease and improved outcome. Bone Marrow Transplant 26: 769-774.

6. Jeha S, Pei D, Raimondi SC, Onciu M, Campana D, et al. (2009) Increased risk for CNS relapse in pre-B cell leukemia with the t(1;19)/TCF3-PBX1. Leukemia 23: $1406-1409$.

7. Huck K, Laws HJ, Meisel R, Traeger A, Bernbeck B, et al. (2006) Three cases of renal relapse after allogeneic hematopoietic stem cell transplantation for childhood acute lymphoblastic leukemia. Haematologica 91: ECR07.
8. Detrait M, Meunier C, Roy DC, Kiss T, Lachance S, et al. (2010) Late acute renal failure due to bilateral kidney infiltration by ALL as single manifestation of relapse after allogeneic transplantation. Bone Marrow Transplant 45: 953-954.

9. Berthou C, Leglise MC, Herry A, Balcon D, Hardy E, et al. (1998) Extramedullary relapse after favorable molecular response to donor leukocyte infusions for recurring acute leukemia. Leukemia 12: 1676-1681.

10. Saito B, Nakashima H, Ariizumi H, Maeda T, Hattori N, et al. (2011) [Bilateral renal infiltration of acute lymphoblastic leukemia cells at relapse after allogeneic stem cell transplantation]. Rinsho Ketsueki 52: 1882-1887.

11. Lankester AC, Bierings MB, van Wering ER, Wijkhuijs AJ, de Weger RA, et al. (2010) Preemptive alloimmune intervention in high-risk pediatric acute lymphoblastic leukemia patients guided by minimal residual disease leve before stem cell transplantation. Leukemia 24: 1462-1469.

12. Sato A, Imaizumi M, Chikaoka S, Niizuma H, Hoshi Y, et al. (2004) Acute renal failure due to leukemic cell infiltration followed by relapse at multiple extramedullary sites in a child with acute lymphoblastic leukemia. Leuk Lymphoma 45: 825-828.

13. Kurts C, Klebba I, Davey GM, Koch KM, Miller JF, et al. (2001) Kidney protection against autoreactive CD8(+) T cells distinct from immunoprivilege and sequestration. Kidney Int 60: 664-671.

14. Crazzolara R, Kreczy A, Mann G, Heitger A, Eibl G, et al. (2001) High expression of the chemokine receptor CXCR4 predicts extramedullary organ infiltration in childhood acute lymphoblastic leukaemia. Br J Haematol 115: 545-553. 